\title{
v-mWater: \\ an e-Government Application for Water Rights Agreements
}

Pablo Almajano, Tomas Trescak, Marc Esteva,

Inmaculada Rodriguez, and Maite Lopez-Sanchez

\begin{abstract}
Nowadays, governments are increasingly taking advantage of Information and Communication Technologies to provide services over the internet (the so-called e-Government applications) to citizens, businesses, employees, and agencies. We argue that e-government services will benefit from being distributed and intelligent, and thus, that they can be modelled as Multi-Agent Systems (MAS). The field of MAS focuses on the design and development of systems composed of autonomous entities which interact within an environment in order to achieve their common or individual goals. Nevertheless, although humans can be seen as autonomous entities, most MAS methodologies and infrastructures do not consider direct human participation. In general, human role is limited to acting behind the scenes by customising provided agent templates. The resulting agents participate in the system on humans' behalf. In order to overcome this limitation we propose using 3D Virtual Worlds, which is one of a very few technologies that provides all the necessary means for direct human inclusion into software systems. 3D Virtual Worlds are 3D graphical environments where humans participate represented as graphical embodied characters (avatars) and can interact there by using simple and intuitive control facilities. We advocate that 3D Virtual Worlds technology can be successfully used for "opening" multiagent systems to humans. This idea is taken in Virtual Institutions, which combine Electronic Institutions and 3D Virtual Worlds to engineer applications where participants may be human and software agents.

In this paper we present the prototype $v$-mWater, a virtual market based on trading Water. It is an e-Government application in the agriculture domain modelled as a Virtual Institution where participants are irrigators and employees of a hydrographic
\end{abstract}

Pablo Almajano · Tomas Trescak · Marc Esteva

Artificial Intelligence Research Institute (IIIA) CSIC, Barcelona (Spain),

e-mail: palmajano,ttrescak,marc@iiia.csic.es

Inmaculada Rodriguez · Maite Lopez-Sanchez

University of Barcelona, Barcelona (Spain),

e-mail: inma,maite@maia.ub.es 
basin. We present the specification of the system, the virtual world generation from this specification and its deployment using the Virtual Institution eXEcution Environment (VIXEE). Finally, we show an example execution of our virtual market in order to illustrate the advantages of our approach.

\section{Introduction}

e-Government is the use of information and communication technologies (ICT) with the aim of providing government services over the internet to citizens, businesses, employees and agencies (e.g. tax returns, virtual offices or help desk applications) [2]. We argue that $e$-Government applications can take advantage of Organisation Centred Multiagent Systems (OCMAS) to model these services as structured interactions between stakeholders and to enforce government norms [16][13]. In particular we are interested in those systems where participants can be both humans and software agents.

Virtual Institutions (VI) combine Electronic Institutions (an OCMAS) and Virtual Worlds technologies [12][4]. They represent 3D virtual spaces where both human and software agents can interact. They offer interesting possibilities to both MAS and 3D virtual environments [7]. First, thanks to the regulation imposed by an OCMAS -in our case an Electronic Institution (EI) [3]-, the 3D environment becomes a normative virtual world where norms are enforced at runtime. Second, a 3D real-time representation of the system allows humans to participate in MAS by controlling its 3D representation (avatar) in an immersive environment. We advocate that VIs can enhance the participation of citizens and business representatives in $e$-Government applications with respect to traditional web-based user interfaces (WUI) or 2D graphical user interfaces (GUI).

In this paper we show an e-Government application for the negotiation of water rights. Governments are employing water markets with the objective of encouraging more efficient use of water for irrigation, above all, in countries with water scarcity problems (e.g. Australia [5]). Our virtual market based on trading Water ( $v$-mWater) is modelled as a VI and facilitates human participation (in our case, citizens and business representatives). It is a simplification of mWater [15] and has been deployed using VIXEE, a robust VI eXEcution Environment that provides interesting features such as multi-verse communication and dynamic manipulation of the virtual world content [22]. VIXEE is a generic and domain-independent solution. Although $v$-mWater is an e-Government application, VI can also be used in other domains which may benefit from structured interactions and norms enforcement such as e-Learning and e-Commerce [8][7].

This paper is structured as follows. First, section 2 provides some background concepts. Second, section 3 specifies $v$-mWater model. Next, section 4 explains the used infrastructure. Afterwards, section 5 discusses the engineering process and shows an example execution. Then, section 6 provides some related work. Finally, section 7 draws the conclusions and propose the future work. 


\section{Background}

\section{Electronic Institutions}

Organisation Centred MAS (OCMAS) approaches are MAS whose foundation lies in organisational concepts [13]. Electronic Institution (EI) is a particular OCMAS that we have used in our application. EIs structure agent interactions by establishing what actions agents are permitted and forbidden to perform as well as their consequences [12]. In particular, interactions are grouped in several dialogic activities (also referred as scenes) where agents participate enacting different roles. Interactions for each activity follow well-defined protocols which are specified by directed graphs whose nodes represent the states and the arcs are labelled with illocution schemes (i.e. events defined as messages) or time-outs. In an activity, participants may change over time, agents may enter or leave.

The so-called performative structure defines how agents can legally move among activities depending on their role. It also defines if an activity can be executed several times at run time and when its execution starts. Specifically, a performative structure is specified as a graph where the nodes represent both activities and transitions - i.e. activity connectives - linked by directed arcs.

\section{Virtual Worlds}

Virtual worlds (VW) are three-dimensional (3D) social spaces where people interact by controlling embodied characters [4] [18]. One of their main features is the immersive experience provided to their participants. They can walk around the world to explore it as done in real spaces. Moreover, they can also fly or even teleport to other places in the VW. Participants interact by using multi-modal communication such as text-based interfaces (e.g. chat windows), voice chat (e.g. using headsets and microphones) or actions performed by avatars (e.g. doing gestures or touching objects). Moreover, the immersive experience can be still increased by incorporating sounds (e.g. birds singing in a virtual forest). Furthermore, they can provide an intuitive graphical representation of the progress of activities that participants are engaged in.

\section{Virtual Institutions}

Virtual Institutions (VI) combine EIs to regulate the participants' interactions and VWs to facilitate human participation in the institution [7]. This way, humans participate in the system by controlling an avatar in the VW, while software agents are directly connected to the EI and can be displayed as bots in the VW to emphasize their artificial nature.

Both EI and VW are causally connected because whenever one of them changes, the other one changes in order to maintain a consistent state [17]. Notice that EI 
and VI have a conceptual difference. EIs define what is permitted and the rest is prohibited. On the contrary, in VIs, only those actions in the virtual world platform that have institutional meaning are regulated, while everything else is permitted.

\section{3 v-mWater Model}

The virtual market based on trading Water ( $v$-mWater) is a VI which models an electronic market of water rights. This market is a simplification of mWater which is an Electronic Institution (EI) focusing on a general water market that includes conflict resolution features (see previous chapter). While mWater includes generic water uses such as human consumption or industrial, we restrict our model to water trading for agricultural purposes, where irrigators are the only actors using the water.

\subsection{Water Market}

Some governments are using markets to regulate the consumption of water from their managed water resources. As previously introduced in section 2.1 of chapter " $m$ Water, a Case Study for Modeling Virtual Markets", in water markets, the goods to negotiate are water rights and the traders are the right-holders. The result of a negotiation is an agreement where a seller agrees to reallocate (part of) the water from her/his rights to a buyer for a fixed period of time in exchange for a certain amount of money.

We model our market in the agriculture domain. More specifically, we consider farmlands that irrigate from water resources totally controlled by public governments. Assigned water rights in this domain are associated to the farmlands. The right-holders are either the owners or the lessees of the farmlands, namely, the irrigators. At the beginning of the irrigation season, the authorities estimate the water reserves and assign the quantity of water to the rights. Tradable water rights ${ }^{1}$ contain the surplus of water the irrigators expect to have on their assigned water rights and decide to sell them. We define an irrigation area as a group of farmlands which can irrigate from the very same water resource -e.g. a reservoir of a basin-. We assume that one farmland only belongs to one area.

Our market opens at the beginning of the irrigation season. Only those irrigators holding rights are allowed to join it. We group the negotiations of water rights by irrigation areas. That means all requested rights' trades of an area are negotiated in the same activity under the same negotiation protocol. Only irrigators holding rights in this area can participate in the negotiation. Moreover, in order to avoid speculation, it is not permitted to resale rights. In order to prevent monopolist strategies, the authorities may establish a maximum water quantity that one irrigator is allowed

${ }^{1}$ From now on, we will refer to these as water rights. 


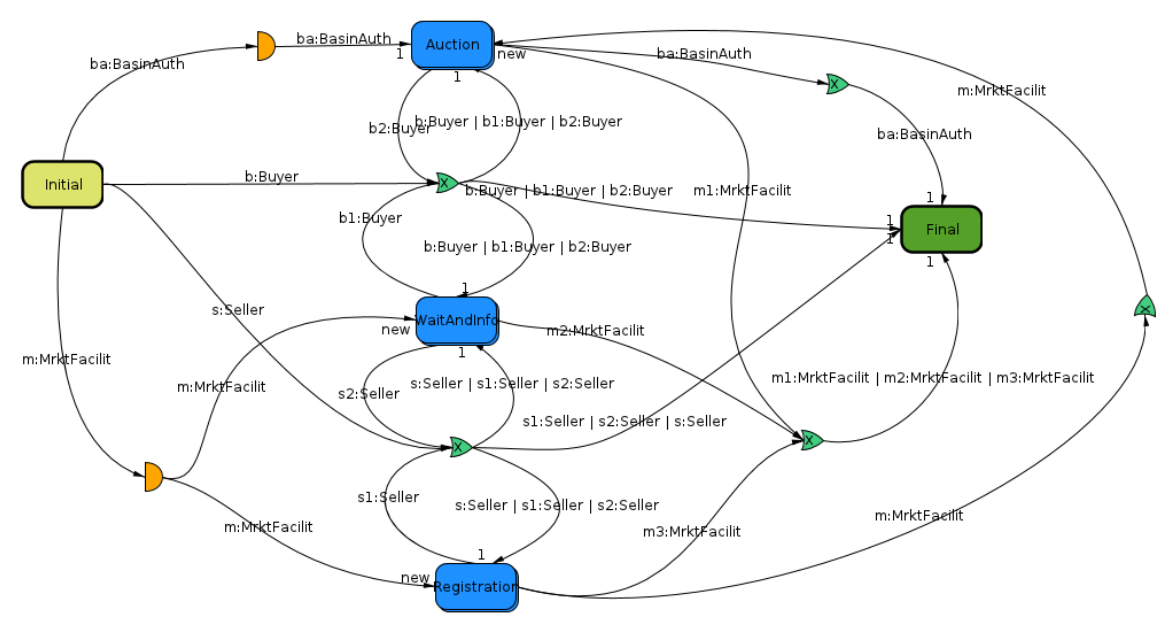

Fig. 1 v-mWater Performative Structure

to buy in a particular area. For example, we can consider a norm such as "one irrigator only can buy a maximum of the $40 \%$ of the total amount of water under negotiation".

\subsection{EI Specification}

From the market defined in previous section 3.1 and using ISLANDER, the EIs specification editor [12], we have defined (1) the ontology of the application, (2) the roles played by participants, (3) the activities involved in the market and the transitions between them (i.e. the performative structure), and (4) the protocols enacting such activities.

The ontology specifies domain concepts such as water right, land, area or agreement. With respect to the roles, agents may adopt a number of them. Irrigator agents can participate as either buyer or seller subroles while market facilitator and basin authority correspond to staff agents. Figure 1 shows the performative structure of $v$-mWater [1]. Besides the obligated initial and final activities to enter and exit the institution, it has three activities which enact the market: Registration, Waiting and Information and Auction. The market facilitator is the responsible of starting the execution of every activity. The basin authority is only allowed to enter to the Auction activity to validate the results. Seller participants can move from the Registration to the Waiting and Information activity and the other way around. On the other hand, buyer agents movements are restricted between Waiting and Information and Auction activities. 


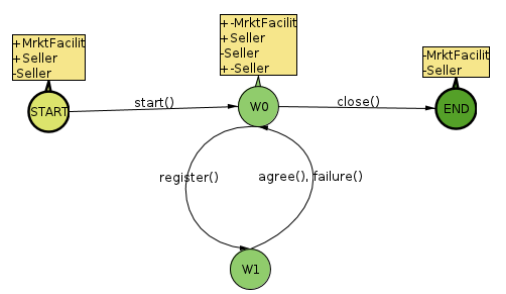

(a) Registration Protocol

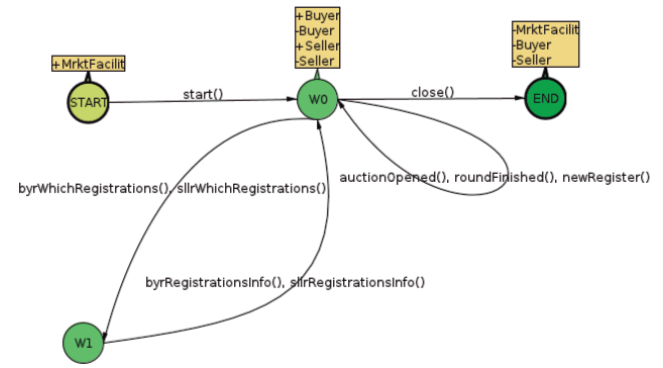

(b) WaitAndInfo protocol

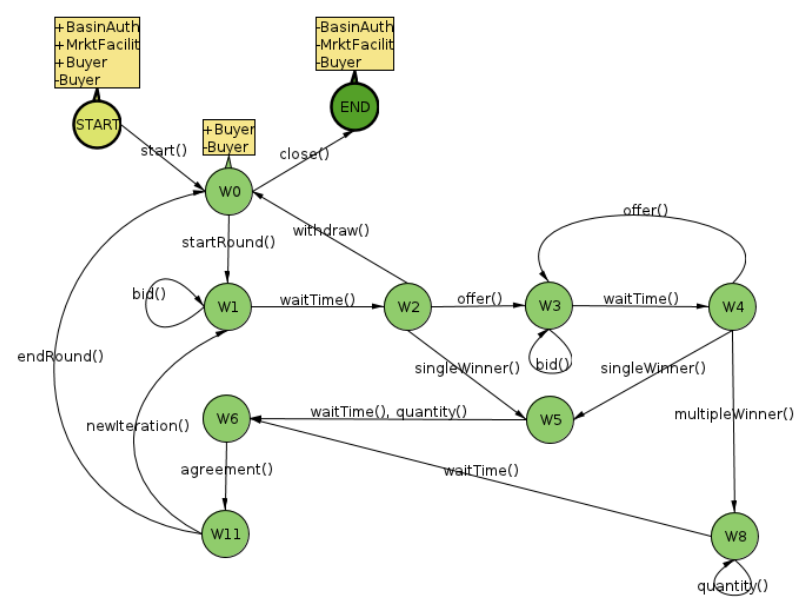

(c) JapaneseAuction protocol

Fig. 2 Activities' protocols: a) Registration; b) Waiting and Information; c) Auction

\section{Registration}

In this activity the market facilitator is in charge of registering sellers' rights. The interactions between participants are regulated following the protocol represented in Figure 2(a). First, a seller asks for registering a right indicating the water quantity to trade. Second, the market facilitator checks whether it is valid or not. Finally, 
the seller is informed about the result of the process (i.e. with an agree or failure message).

\section{Waiting and Information}

This activity follows the protocol depicted in Figure 2(b). It permits irrigators (buyers and sellers) to request information about negotiations to the market facilitator. Moreover, all participants within the activity are proactively informed when: i) a new auction has been opened, so buyers are able to enter; ii) an auction round is finished, informing about reached agreements; and iii) a seller has successfully registered a new right that will be negotiated later on in the corresponding auction activity. Therefore, sellers can wait within this activity for the result of the negotiations of their rights after registering them, and buyers can wait until the auction they are interested in opens.

\section{Auction}

The negotiation of water rights takes place in this activity. There are three roles involved in it. The market facilitator conducts the auction, buyers bid for water rights and the basin authority announces the valid agreements. All (previously registered) rights belonging to the same area are negotiated within the same activity. Therefore, one auction activity is created for each area that has available water rights to negotiate.

A multi-unit Japanese auction protocol enacts the activity (see Figure 2(c)). In this protocol, registered water rights -composed by several litres of water- are auctioned in consecutive rounds, i.e one round per registered water right. Buyers only can join and leave the auction between rounds. Market facilitator starts a new round at a previously established price. It is divided in several iterations following these four rules: (1) the price goes up in regular increments; (2) only buyers that bid at previous increment are allowed to place bids (all of them in case of first increment) (3) the iteration ends when i) just one buyer bids at current increment (single winner) or ii) no bids are performed, so the winners are the buyers that bid at previous increment; (4) winner(s) request the amount of water desired. If there is more than one winner, then the water is assigned by following a proportional allocation algorithm. Once an iteration is finished, the basin authority validates the result(s) -winner(s) have requested a minimum quantity of water and have enough creditand announces the agreement(s). The round ends either when there was no bid in last iteration or the water right under negotiation has no more water available. The negotiation is over when all rights have been traded.

The activities explained above have the following correspondences with $m$ Water Performative Structures (PS) defined in section 2.3 of previous chapter: (1) Registration is a simplification of Accreditation; (2) in Waiting and information, water 


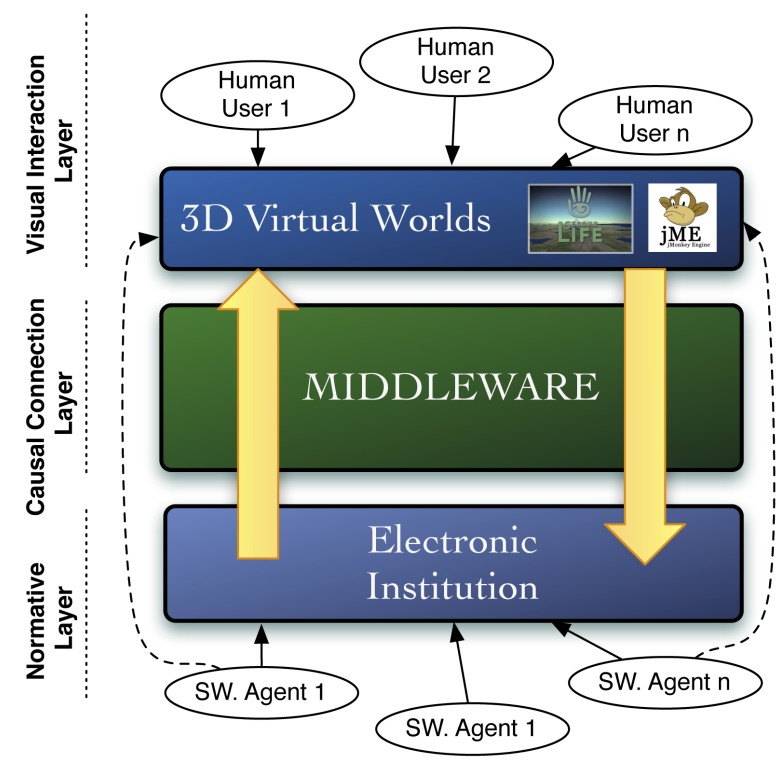

Fig. 3 Overview of VIXEE Architecture

users may obtain information about negotiations as in Open Trades and Ongoing Agreements -both located in the TradingHall PS-; and (3) Auction activity includes Agreement Validation as well as a particular trading protocol of the TradingTable $P S$.

\section{VIXEE Architecture}

We have deployed $v$-mWater model using the Virtual Institution eXEcution Environment (VIXEE) [22]. Figure 3 depicts its architecture composed of three layers: i) normative, ii) visual interaction and iii) causal connection.

The normative layer is composed by AMELI, the electronic institutions infrastructure that mediates agents' interactions while enforcing institutional rules [12]. AMELI can be regarded as domain-independent because it can interpret any institution specification generated by ISLANDER tool [12]. In our case, it interprets the specification defined in section 3.2. It is implemented in JAVA and uses two TCP ports for communication with the causal connection layer.

The visual interaction at the top layer comprises several 3D virtual worlds. Each Virtual World (VW) can be implemented in a different programming language using a different graphics technology. The usual parts of a VW are a VW client and a VW server. Such a server communicates with the causal connection layer using a 
standard protocol (e.g. UDP, TCP or HTTP). In our application we employ Open Simulator, an open source multi-platform, multi-user 3D VW server [19].

The causal connection layer causally connects the visual interaction and the normative layers, i.e. whenever one of them changes, the other one changes in order to maintain a consistent state [17]. This layer implements a multi-verse communication mechanism that allows users from different virtual worlds to participate in the same VI. The mapping between VW actions and AMELI protocol messages -and vice versa- is defined by a movie script mechanism. Moreover, VIXEE uses the Virtual World Grammar (VWG) mechanism and its implementation in the Virtual World Builder Toolkit (VWBT) to dynamically manipulate the 3D representation of all connected virtual worlds [21].

\section{Setting up the Model}

In order to engineer $v$ - $m$ Water, we define the three following steps:

First, we specify the normative control layer of the virtual institution -that is an electronic institution- using ISLANDER tool [12]. The output is the electronic institution specification introduced in section 3.2.

Second, using the VWBT tool, we generate the 3D representation from $v$-mWater specification. Figure 4(a) depicts the resulted generation in Open Simulator [19]. In particular, it shows an aerial view of three rooms located at an open space that correspond to the three main activities in $v$-mWater. Participants join and leave these activities by opening (and crossing) the doors of these rooms. Moreover, transitions between activities are experienced as movements in the open space.

Third, using the movie script mechanism we define the mapping between VW actions and EI messages and vice versa. In this first prototype, some actions in the VW (such as touching a door to open it) are mapped to EI messages (join the activity taking place in the room). Additionally, commands typed on chat windows in the VW (e.g., the login chat represented in Figure 4(b)) have been mapped to protocol messages in the EI. On the other hand, some of the bot messages in the EI are represented as gestures made by its respective avatar in the VW. Thus, for instance, a "bid" message is mapped to a "raise hand" gesture as depicted in Figure 4(d)).

\subsection{Running the application}

In this section we comment on key aspects of the result of the engineering process mentioned above. They are introduced by following a particular sequence that a given participant may follow ${ }^{2}$.

\footnotetext{
${ }^{2}$ Watch video at youtube http://www.youtube.com/watch?v=OisCys8q_i8 for a complete visualization of such a participation sequence
} 


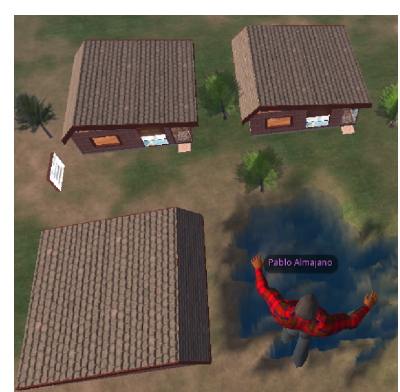

(a) Initial aerial view

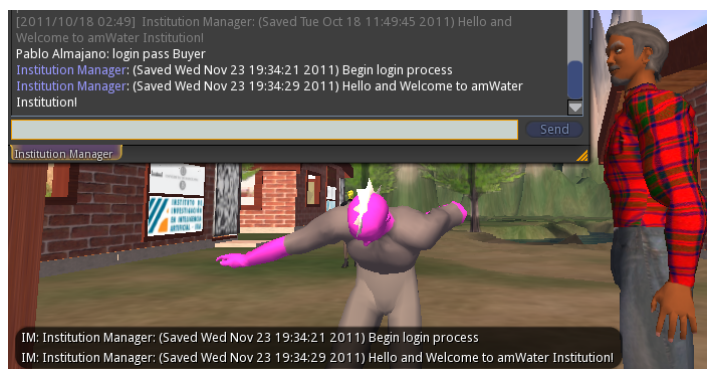

(b) Human avatar login: interaction with a software agent by means of a chat window

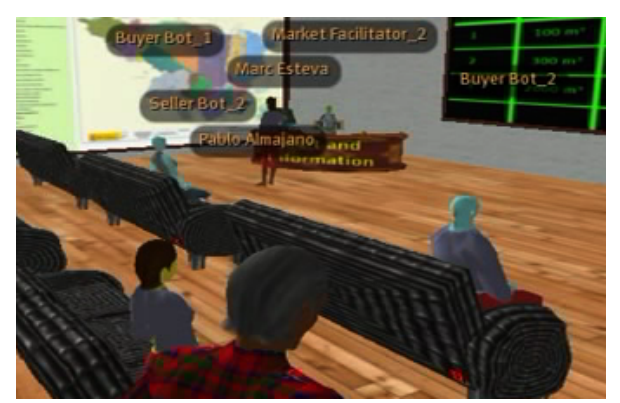

(c) The inside of the Waiting and Information room

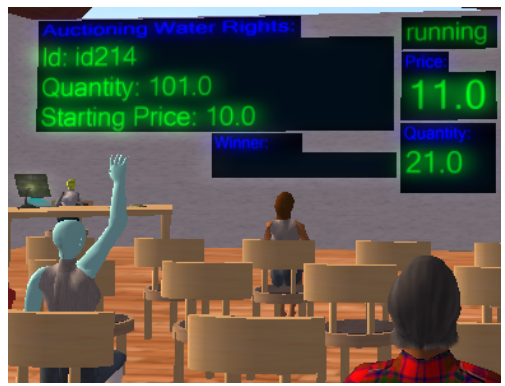

(d) Bot bidding in a running auction

Fig. 4 Examples of $v$-mWater running

Nevertheless, before getting into the steps, it is worth noticing that software agents have been characterized as bots with the aim of enhancing their artificial nature: they are bold and have differentiated artificial skin colours that represent their roles (see Figures 4(b), (c) and (d)).

In order to Login in the institution, we send a private message to the Institution Manager bot with the content "login pass role". Where "pass" is replaced by our password and "role" by the desired role to play in the institution (either seller or buyer). The welcome event to the institution has been mapped to a "greeting" gesture made by the Institution Manager avatar (see Figure 4(b)).

When playing a seller role, we can register a water right in the Registration room by sending the "register" command privately to the market facilitator which is sat at a desktop. This command includes the quantity of water to negotiate. The market facilitator then performs the registering process and sends us back an "ok" or "failure" message.

We can access the Waiting and Information room (depicted in Figure 4(c)) by enacting a seller or a buyer role. In this room, we can ask for information about negotiations to the market facilitator sat at a desktop. Furthermore, we can wait by sitting down on the sofas arranged in the room and consult the available information about negotiations displayed on the dynamic information panels. 
In the Auction room the market facilitator and the basin authority bots are sited at their respective desktops and several chairs are disposed within the room for buyer participants. Figure 4(d) shows how human participation in the auction has been improved by providing a comprehensive environment that includes dynamic information panels. Moreover, bots bid actions can be also easily identified by human participants since they are displayed as raising hands.

\section{Related Work}

Public administrations are increasing the use of e-Governments to provide a variety of services over the internet to citizens such as, for instance, tax returns, administrative process, personal information update and voting [9]. A water market is a government service available in many countries with water scarcity problems that can be provided as an e-Government application. For instance, Waterfind is an intermediary private company which offers web-based tools to access the national market in Australia (e.g., place online buy or sell water rights orders, buy from or sell to a previous registered order or see real-time information about orders) [23]. $v$ $m$ Water can provide such a tools (because it is an electronic market for water rights) and also improve human participation by means of an interactive and immersive 3D environment.

A research work models a MAS for the management of e-Government services. It improves citizens' access to government information distributed among agencies following strict interactions [11]. $v$-mWater is modelled as an OCMAS which structures agent interactions as well as enforce government norms.

With respect to MAS and VW combination, Ranathunga et al provide a framework which connects a BDI agent platform to the VW server Second Life ${ }^{3}$ [20]. This framework includes an online monitor of social expectations that notifies agents when their expectations of others (i.e. actions performed in the virtual world) have been fulfilled or violated [10]. Another work integrates a MAS developed in JADE with the VW server Open Wonderland (http://openwonderland.org/) by modifying an existing Open Wonderland module that starts a JADE agent [6]. v-mWater is a Virtual Institution that uses a robust infrastructure which causally connect a domain independent OCMAS platform (EI) with VWs.

Gartner et al. combine an EI and a VW to deploy an e-Commerce virtual organisation in the tourism domain by using the 3D Electronic Institution framework architecture [14]. Regarding Virtual Institutions, an e-Learning application simulates the culture of the ancient City of Uruk [8]. Both infrastructures allow the connection of an EI to a given VW, while $v$-mWater uses VIXEE architecture that allows the connection to multiple VWs as well as the dynamic manipulation of the 3D representation of all connected VWs.

${ }^{3}$ http://secondlife.com/ 


\section{Conclusions and Future Work}

In this paper we use Virtual Institutions (VI), which combine both multi-agent systems and 3D interfaces, to engineer e-* applications. A VI is composed of i) a normative layer which structures participants interactions, ii) a visual interaction layer which provides a 3D interface for direct human participation in the system, iii) and a communication layer.

We present an e-government prototype for water rights agreements named $v$ $m$ Water, a virtual market based on trading Water. First, we specify the normative layer, i.e the Electronic Institution (EI) which defines agent roles, activities' protocols and roles' workflow between activities. After, from this specification we generate the visual interaction layer, i.e a 3D Virtual World (VW) representation using the Virtual World Builder Toolkit (VWBT). And finally, we use the Virtual Institution eXEcution Environment (VIXEE) to deploy $v$-mWater, connecting the normative (i.e EI) and the visual interaction (i.e VW) layers.

We proposed an immersive environment where humans participate in the institution by controlling an avatar which allows their interaction with the environment and other participants, software or human agents. Software agents are directly connected to the institution and can be represented as bots in the VW in order to highlight their artificial nature. As result, our system has favoured direct human participation in MAS.

As future work, we will extend $v$-mWater with assistance services to participants, and so make more efficient their participation in the system. Moreover, we plan to evaluate the usability of the prototype by measuring interface effectiveness, efficiency and user experience.

Acknowledgements This work is partially funded by EVE (TIN2009-14702-C02-01 / TIN200914702-C02-02) and AT (CONSOLIDER CSD2007-0022) Spanish research projects, EU-FEDER funds and the Catalan government (Grant 2005-SGR-00093).

In memoriam of Marc Esteva, who actively contributed to our research line. With his passing we have lost a great colleague and supervisor. It will be an honour to continue working on his scientific seed.

\section{References}

1. P. Almajano, M. Lopez-Sanchez, M. Esteva, and I. Rodriguez. An assistance infrastructure for open mas. volume 232, pages 1-10, Lleida, Catalonia, Spain, 26/10/2011 2011. IOS Press.

2. T. Almarabeh and A. AbuAli. A general framework for e-government: Definition maturity challenges, opportunities, and success. European Journal of Scientific Research, 39(1):29-42, 2010.

3. J. Arcos, M. Esteva, P. Noriega, J. Rodríguez-Aguilar, and C. Sierra. An integrated development environment for electronic institutions. In Software Agent-Based Applications, Platforms and Development Kits, pages 121-142. 2005.

4. R. Bartle. Designing Virtual Worlds. New Riders Games, 072003. 
5. H. Bjornlund and P. Rossini. Climate change, water scarcity and water markets: Implications for farmers? wealth and farm succession. In Proceedings of the PRRES Conference, 2010.

6. J. Blair and F. Lin. An approach for integrating $3 \mathrm{~d}$ virtual worlds with multiagent systems. In Advanced Information Networking and Applications (WAINA), 2011 IEEE Workshops of International Conference on, pages 580 -585, march 2011.

7. A. Bogdanovych. Virtual Institutions. PhD thesis, University of Technology, Sydney, Australia, 2007.

8. A. Bogdanovych, J. A. Rodriguez-Aguilar, S. Simoff, and A. Cohen. Authentic interactive reenactment of cultural heritage with $3 \mathrm{~d}$ virtual worlds and artificial intelligence. Appl. Artif. Intell., 24:617-647, July 2010.

9. A. Chadwick and C. May. Interaction between states and citizens in the age of the internet: egovernment in the united states, britain, and the european union. Governance, 16(2):271-300, 2003.

10. S. Cranefield and G. Li. Monitoring social expectations in second life. In Proceedings of The 8th International Conference on Autonomous Agents and Multiagent Systems - Volume 2, AAMAS '09, pages 1303-1304, Richland, SC, 2009. International Foundation for Autonomous Agents and Multiagent Systems.

11. P. De Meo, G. Quattrone, D. Ursino, and G. Terracina. A multi-agent system for the management of e-government services. In Proceedings of the IEEE/WIC/ACM International Conference on Intelligent Agent Technology, IAT '05, pages 718-724, Washington, DC, USA, 2005. IEEE Computer Society.

12. M. Esteva. Electronic institutions. from specification to development. $\mathrm{PhD}$ thesis, UPC, 2003.

13. J. Ferber, O. Gutknecht, and F. Michel. From agents to organizations: An organizational view of multi-agent systems. In Agent-Oriented Software Engineering IV, volume 2935, pages 443-459. 2004.

14. M. Gärtner, I. Seidel, J. Froschauer, and H. Berger. The formation of virtual organizations by means of electronic institutions in a 3d e-tourism environment. Inf. Sci., 180:3157-3169, September 2010.

15. A. Giret, A. Garrido, J. A. Gimeno, V. Botti, and P. Noriega. A mas decision support tool for water-right markets. In AAMAS '11, pages 1305-1306, 2011.

16. N. R. Jennings, K. Sycara, and M. Wooldridge. A roadmap of agent research and development. Autonomous Agents and Multi-Agent Systems, 1:7-38, 1998.

17. P. Maes and D. Nardi, editors. Meta-Level Architectures and Reflection. Elsevier Science Inc., NY, USA, 1988

18. P. R. Messinger, E. Stroulia, K. Lyons, M. Bone, R. H. Niu, K. Smirnov, and S. Perelgut. Virtual worlds - past, present, and future: New directions in social computing. Decision Support Systems, 47(3):204-228, June 2009.

19. OpenSimulator. http://opensimulator.org, 2011.

20. S. Ranathunga and S. Cranefield. Interfacing a cognitive agent platform with second life. Information Science Discussion Papers Series, 032011.

21. T. Trescak, M. Esteva, and I. Rodriguez. A virtual world grammar for automatic generation of virtual worlds. The Visual Computer, 26:521-531, 2010.

22. T. Trescak, M. Esteva, and I. Rodriguez. Vixee an innovative communication infrastructure for virtual institutions (extended abstract). In AAMAS '11, pages 1131-1132, 2011.

23. WaterFind. Water market specialists, http://www.waterfind.com.au/, 2011. 